\title{
THE CRYOGENIC DISTRIBUTION LINE FOR THE LHC: FUNCTIONAL SPECIFICATION AND CONCEPTUAL DESIGN
}

\author{
W. Erdt, G. Riddone and R. Trant
}

\begin{abstract}
The Large Hadron Collider (LHC)1 currently under construction at CERN will make use of superconducting magnets operating in superfluid helium below $2 \mathrm{~K}$. The cryogenic distribution scheme for each of the eight sectors, individually served by a refrigeration plant, is based on a separate Cryogenic Distribution Line (QRL) feeding helium at different temperatures and pressures to the elementary cooling loops. The QRL comprises two supply headers and three return headers including a sub-atmospheric one. Low heat inleak to all temperature levels is essential for the overall LHC cryogenic performance. With an overall length of $25.6 \mathrm{~km}$ the QRL has a very critical cost-to-performance ratio. Therefore, following an in-house feasibility study, CERN adjudicated in autumn 1998 three industrial contracts in parallel for the supply of Pre-Series Test Cells ( 112 m) of the QRL, which will be tested at CERN in 2000. Installation of the QRL for LHC is scheduled from 2002 to mid 2004. This paper will present the general layout, the functional requirements as well as some aspects of the in-house conceptual design.
\end{abstract}

LHC Division

Presented at the 1999 Cryogenic Engineering and International Cryogenic Materials Conference

(CEC-ICMC'99), 12-16 July 1999, Montreal, Canada

Administrative Secretariat

LHC Division

CERN

CH - 1211 Geneva 23

Switzerland

Geneva, 1 December 1999 


\title{
THE CRYOGENIC DISTRIBUTION LINE FOR THE LHC: FUNCTIONAL SPECIFICATION AND CONCEPTUAL DESIGN
}

\author{
W. Erdt, G. Riddone, R. Trant \\ CERN, LHC Division \\ CH-1211 Geneva 23, Switzerland
}

\begin{abstract}
The Large Hadron Collider (LHC) ${ }^{1}$ currently under construction at CERN will make use of superconducting magnets operating in superfluid helium below $2 \mathrm{~K}$. The cryogenic distribution scheme for each of the eight sectors, individually served by a refrigeration plant, is based on a separate Cryogenic Distribution Line (QRL) feeding helium at different temperatures and pressures to the elementary cooling loops. The QRL comprises two supply headers and three return headers including a sub-atmospheric one. Low heat inleak to all temperature levels is essential for the overall LHC cryogenic performance. With an overall length of $25.6 \mathrm{~km}$ the QRL has a very critical cost-to-performance ratio. Therefore, following an in-house feasibility study, CERN adjudicated in autumn 1998 three industrial contracts in parallel for the supply of Pre-Series Test Cells $(\sim 112 \mathrm{~m})$ of the QRL, which will be tested at CERN in 2000. Installation of the QRL for LHC is scheduled from 2002 to mid 2004. This paper will present the general layout, the functional requirements as well as some aspects of the in-house conceptual design.
\end{abstract}

\section{INTRODUCTION}

The LHC cryogenic system ${ }^{2}$, shown in Figure 1, is based on a five-point feed scheme with eight refrigeration plants serving the eight sectors of the LHC machine. Each of the eight sectors $(\sim 3.3 \mathrm{~km})$ has its own self-standing cryogenic system. The cryogen provided by a refrigeration plant of $18 \mathrm{~kW}$ at $4.5 \mathrm{~K}$ equivalent power ${ }^{3}$, is distributed at different temperatures and pressures via the Cryogenic Distribution Line $(\mathrm{QRL})$ to the LHC magnet cryostats and other cryogenic consumers of the relevant sector. A $1.8 \mathrm{~K}$ refrigeration unit ${ }^{4}$ maintains, via a large pumping line inside the QRL, the low pressure $(1.6 \mathrm{kPa})$ for the local superfluid helium heat exchangers ${ }^{5}$ inside the cold mass. The LHC cryogenic system will for the first time make use of a cold recovery line, also integrated into the QRL, to collect the helium discharged from the machine cryostats following a quench. The central 
connecting element between all these parts of the cryogenic distribution system of a LHC feeding point is the Cryogenic Interconnection Box.

\section{GENERAL LAYOUT}

Each LHC sector, as shown in Figure 1, is composed of different sections, namely Long Straight Sections (LSS), Dispersion Suppressors (DS) and arc, which constitutes about $3 / 4$ of the total sector length. The QRL structure follows the LHC lattice, which is for the arc made of 23 identical cells, each of $106.9 \mathrm{~m}$ length. The DS is made up of 4 cells of length, which vary from $80 \mathrm{~m}$ to $91.6 \mathrm{~m}$. The LHC lattice in the LSS is very particular in layout for each of these regions with QRL cell lengths from about $10 \mathrm{~m}$ to $170 \mathrm{~m}$.

Each QRL cell consists of a Service Module and a Pipe Module as shown in Figure 2. Different cell lengths are accommodated by adjusting the Pipe Module length. The Service Module comprises two Valve Boxes and the so-called Jumper Connection, by which the QRL is linked to the magnet cryostat. The service module houses a subcooling helium heat exchanger, up to seven cryogenic control valves, one or two superfluid helium safety relief valves $^{6}$, a cryogenic mass flowmeter ${ }^{7}$ as well as monitoring instrumentation. The Pipe Module is made of several Straight Pipe Elements and Compensation Units.

The cooling loop of a LHC standard cell, as shown in Figure 3, contains Service Modules of Type A and B, the most frequent ones. The QRL headers will supply helium to the LHC magnet cryostat at different temperatures and pressures. Supercritical helium at 4.6 $\mathrm{K}$ and $0.3 \mathrm{MPa}$ tapped from header $\mathrm{C}$ will be used to supply the cold mass HeII heat exchanger and the beam screen circuit as well as for lower temperature heat interceptions and initial filling. The return line is header $\mathrm{D}$ that has also the function of cold recovery line. Helium at $4 \mathrm{~K}$ and very low pressure $(1.6 \mathrm{kPa})$ will circulate in header $\mathrm{B}$ for maintaining the saturation pressure of the superfluid helium circuit. Finally, gaseous helium at 50 to $75 \mathrm{~K}$ will cool the thermal shields of the QRL and the LHC magnet cryostats. Inside the arc and DS the supply header E will be housed inside the LHC magnet cryostats while the return header F will always be integral part of the QRL.

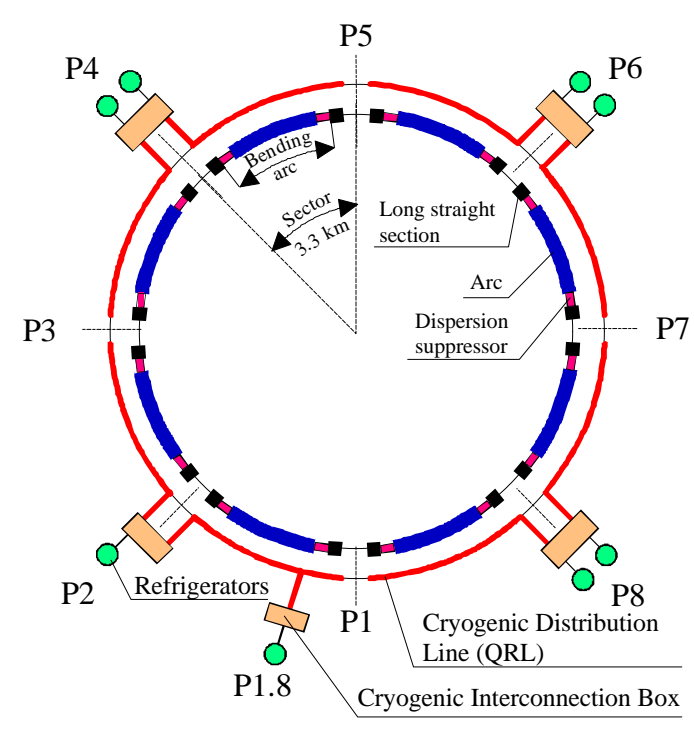

(a)

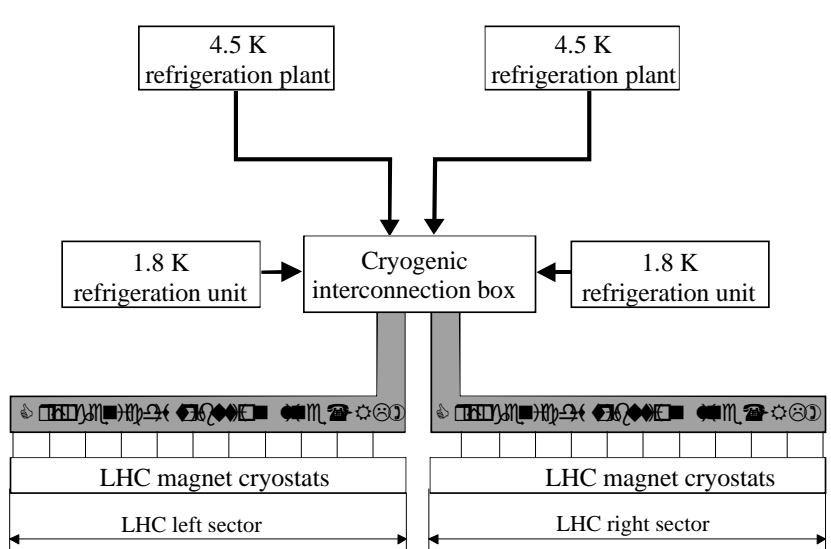

(b)

Figure 1. LHC cryogenic system: (a) layout and (b) architecture of a typical feeding point 


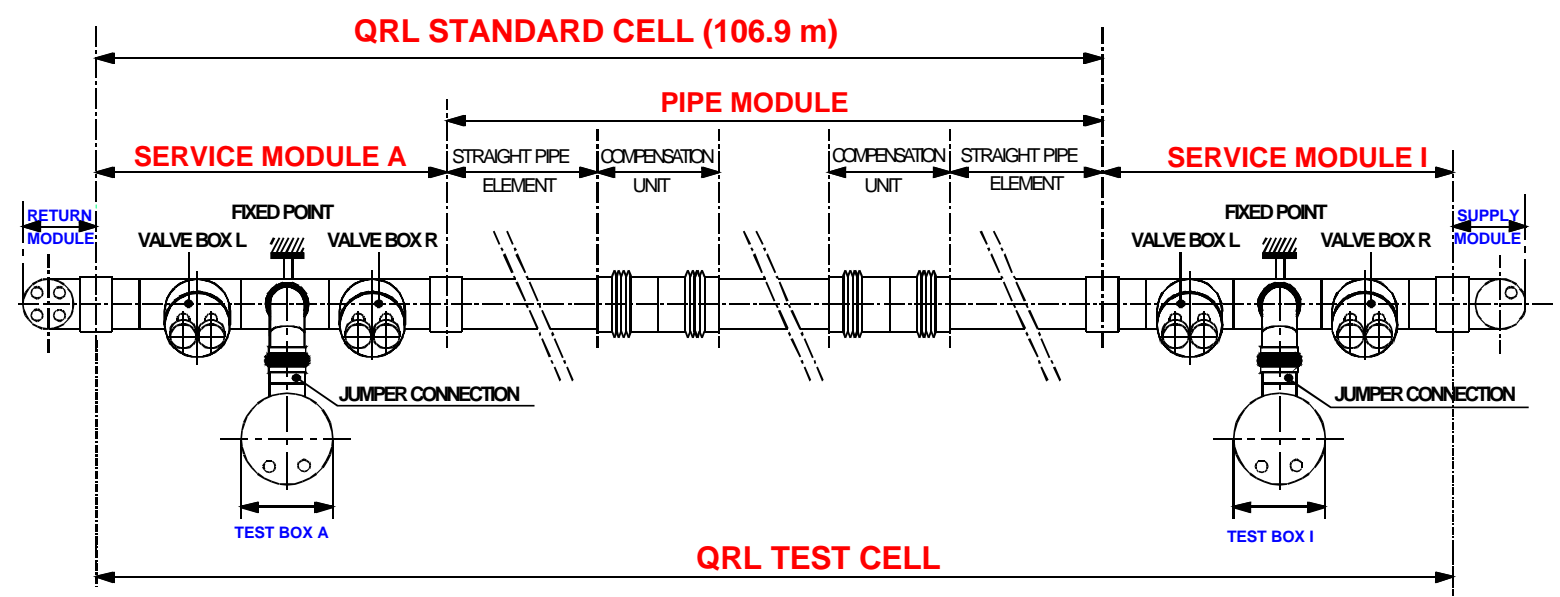

Figure 2. QRL standard cell and test cell (based on CERN feasibility study)

According to the overall LHC cryogenic flow scheme, all cooling loops are similar but not always identical. This leads to 26 different Service Module types supplying the cryogen needed to magnets operating at $1.9 \mathrm{~K}$ or $4.5 \mathrm{~K}$, electrical feed-boxes and superconducting cavities. By minor modifications most of the types can be derived from a basic type.

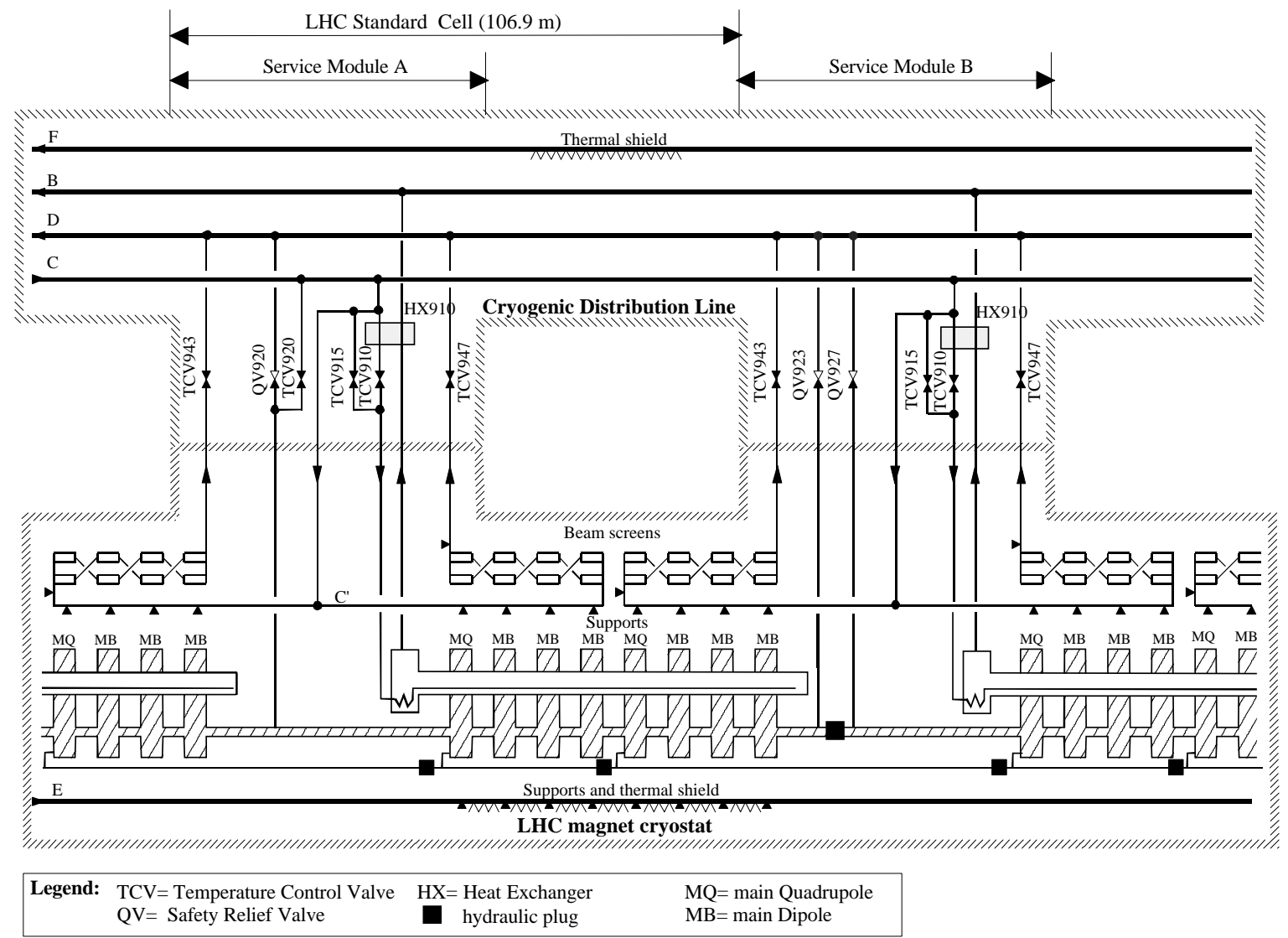

Figure 3. Typical LHC cryogenic flow scheme 


\section{BASIC REQUIREMENTS AND DESIGN CHALLENGES}

The lifetime of the LHC is expected to be 20 years with a yearly operating time of 6600 hours for the cryogenic distribution system ${ }^{8}$. Cryogenic supply is needed all around the ring and tunnel access for maintenance purposes will be limited. Thereby reliability of the cryogenic system becomes very challenging. Furthermore all tunnel equipment must withstand the expected radiation environment ${ }^{8}$.

The QRL design must be highly reliable, which is of first importance for the active components such as bellows and control valves. The cryogenic compensation units for the headers ( 4000) and the metal hoses inside the Jumper Connections ( 4000, see machine interface below) must be designed in accordance with well established international standards and will have to undergo extensive testing and qualification programs. Reliability as well as control and monitoring of the about 1500 cryogenic valves around the tunnel become important issues. CERN considers the use of new generation valve positioners, the so-called "intelligent" positioners, presently under evaluation". They allow for automatic start-up, including self-calibration, remote configuration as well as preventive maintenance planning using diagnostic data.

The QRL design should be independent of the cooling rate, the cool-down sequence of the various headers and the pressurisation rate (e.g. header D in case of a helium discharge from a magnet cryostat following a quench). The nominal QRL operating conditions and required header diameters are listed in Table 1.

With a total length of about $25.5 \mathrm{~km}$ the QRL is a largely modular set-up of Pipe and Service Modules allowing a high degree of standardisation and consequently series production of the various elements. The industrial series production of highly reliable cryogenic modules with unprecedented low heat inleak for the lowest price per meter ever achieved is the real challenge of this project.

\section{THERMAL REQUIREMENTS}

The required heat inleak for a QRL Standard Cell (header E is not included) and the QRL sectors (headers are grouped with reference to the reception test scheme) are given in Table 2. The values are based on a CERN design and feasibility study. Considering the overall LHC thermal budget ${ }^{10}$ on the different temperature levels, the heat inleaks of the QRL contribute for about $60 \%$ to $50-75 \mathrm{~K}$ (headers $\mathrm{E}$ and F), $5 \%$ to $4.6-20 \mathrm{~K}$ (headers $\mathrm{C}$ and D) and $95 \%$ to $4 \mathrm{~K}$ (header B).

To limit the heat inleak by radiation from room temperature, the QRL cryostat will house a thermal shield and radiative insulation. The thermal shield, cooled by the return header $\mathrm{F}$ at $75 \mathrm{~K}$, will surround the components which are at temperatures below $50 \mathrm{~K}$. The outer surface of the thermal shield will be wrapped with multilayer insulation (30 layers). The cryogenic headers below $20 \mathrm{~K}$ will be individually or commonly wrapped by a "floating" insulation system (e.g. 10 layers). The choice of multilayer insulation is of crucial importance for meeting the total QRL budget, as at 4-20 K its contribution represents about $3 / 4$ of the total estimated heat inleaks. Indicative values, measured at $\mathrm{CERN}^{11}$, of heat flux through multilayer insulation at $10^{-4} \mathrm{~Pa}$ (nitrogen equivalent measured with a room temperature gauge on the vacuum jacket) are $1 \mathrm{~W} / \mathrm{m}^{2}$ from $300 \mathrm{~K}$ to $75 \mathrm{~K}$ for 30 layers and $0.05 \mathrm{~W} / \mathrm{m}^{2}$ from $75 \mathrm{~K}$ to $5 \mathrm{~K}$ for 10 layers. 
Table 1: QRL headers: diameter \& nominal operating conditions

\begin{tabular}{ccccccc}
\hline Header & Description & $\begin{array}{c}\text { Inner Diameter } \\
{[\mathrm{mm}]}\end{array}$ & $\begin{array}{c}\text { Nominal } \\
\text { Temperature } \\
{[\mathrm{K}]}\end{array}$ & $\begin{array}{c}\text { Nominal } \\
\text { Pressure } \\
{[\mathrm{MPa}]}\end{array}$ & $\begin{array}{c}\text { Design } \\
\text { Pressure } \\
{[\mathrm{MPa}]}\end{array}$ & $\begin{array}{c}\text { Nominal } \\
\text { Mass flow rate } \\
{[\mathrm{g} / \mathrm{s}]}\end{array}$ \\
\hline B & pumping return & 267 & $3.8-4.2$ & 0.0016 & 0.4 & 125 \\
$\mathrm{C}$ & 4.6 K supply & 100 & 4.6 & 0.36 & 2 & 215 \\
$\mathrm{D}$ & 20 K return & 150 & 20 & 0.13 & 2 & 90 \\
E & 50 K supply & 80 & $50-65$ & 1.95 & 2.2 & 250 \\
F & 75 K return & 80 & $65-75$ & 1.9 & 2.2 & 250 \\
\hline
\end{tabular}

The thermal shield and the header assembly will be supported by about 20 sliding and 4 fixed points per QRL Standard Cell. Low thermal conductivity components will have to be used together with heat interceptions to intermediate temperatures.

At working temperature without active pumping the required insulation for each insulation space $(428 \mathrm{~m})$ is $10^{-4} \mathrm{~Pa}$. During nominal LHC operation the insulation vacuum of each of the vacuum spaces will be maintained without continuous mechanical pumping.

\section{INTERFACE TO THE MACHINE CRYOSTATS (JUMPER CONNECTION)}

The high alignment precision of the LHC machine has two implications for the QRL. Firstly the QRL must not introduce significant forces to the machine elements via the Jumper Connection every $106.9 \mathrm{~m}$, which might lead to a misalignment. Therefore the interconnecting process pipes as well as the vacuum jacket must be equipped with flexible elements. Secondly the LEP (Large Electron Positron collider) operation has shown that the long-term stability of the tunnel plane due to geological movements is significantly less than the required alignment precision for the LHC. In order to realign machine elements without moving the QRL, the Jumper Connections must be able to compensate for movements of the beam tubes relative to the QRL of $\pm 10 \mathrm{~mm}$ in the horizontal plane and $\pm 20 \mathrm{~mm}$ in the vertical direction. For accumulated displacements exceeding these values the QRL external supports should give additional adjustment capability.

Table 2: Required heat inleak for (a) QRL Standard Cell (106.9 m) and (b) QRL sectors

(a)

\begin{tabular}{lcccc}
\hline Heat inleaks & $\begin{array}{c}\text { Header B } \\
{[\mathrm{W}]}\end{array}$ & $\begin{array}{c}\text { Header C } \\
{[\mathrm{W}]}\end{array}$ & $\begin{array}{c}\text { Header D } \\
{[\mathrm{W}]}\end{array}$ & $\begin{array}{c}\text { Header F } \\
{[\mathrm{W}]}\end{array}$ \\
\hline Pipe Module $(104.3 \mathrm{~m})$ & 6.4 & 2.5 & 4.0 & 270 \\
Service Module type A $(2.6 \mathrm{~m})$ & 0.7 & 1.6 & 1.2 & 30 \\
\hline Total & 7.1 & 4.1 & 5.2 & 300 \\
\hline \multicolumn{3}{c}{ Headers E and F } & \multicolumn{2}{c}{ Headers C, B and D } \\
\hline Heat inleaks & \multicolumn{2}{c}{$[\mathrm{W}]$} & \multicolumn{2}{c}{520} \\
& \multicolumn{2}{c}{9000} & \multicolumn{2}{c}{580} \\
\hline Low load sectors: $2-3,3-4,6-7,7-8$ & \multicolumn{2}{c}{9850} & \\
High load sectors: $1-2,4-5,5-6,8-1$ & \multicolumn{3}{c}{5} \\
\hline
\end{tabular}




\section{TUNNEL INSTALLATION}

The QRL will be placed inside the LHC tunnel between the machine cryostat and the outer tunnel wall, where the available space is very limited. Figure 4 shows standard tunnel cross-sections with a QRL design based on a CERN in-house feasibility study.

For the QRL with an expected outer diameter of about $610 \mathrm{~mm}$ a maximum outer envelope of $750 \mathrm{~mm}$ is foreseen, which includes available space for sleeves and expansion joints, as well as any kind of tooling like welding/cutting devices. In order to fit the valve boxes to the space constraints and to allow for exchanging stems of the cryogenic control valves, the CERN study foresees a tilt of the valve boxes by 10 degrees towards the machine cryostat.

\section{PRE-SERIES TEST CELL}

The aim of a Pre-series Test Cell is to qualify the design chosen and to verify its thermal and mechanical performance. The engineering of the Pre-series Test Cell fully takes into account the subsequent series production of the basic QRL elements and is as close as possible to the final concept, such as compensation units, multilayer insulation wrapping, installation procedures or polygonal geometry.

The QRL Test Cell will first be used for qualifying the manufacturing process and the validation of installation and quality control procedures. Through extensive testing at CERN, it will then be used to investigate heat inleaks and to perform thermal cycles and pressure tests in order to verify the design with respect to the assumed load breakdown. The test results may give input for simplification and subsequent reduction in cost for the modular QRL elements to be produced in series.

The QRL Test Cell (see Figure 2), approximately $112 \mathrm{~m}$ long, will consist of a QRL Standard Cell and an additional Service Module needed for test purposes (namely Service Module type I). To perform the reception tests as close as possible to the LHC working conditions, each QRL Test Cell will be individually fitted with the corresponding ancillary equipment such as a test supply module, a test return module and two test boxes. This test equipment will be connected to the Test Cell in the same way as the QRL sub-assembly interfaces and the future Jumper Connection to the LHC machine cryostats.

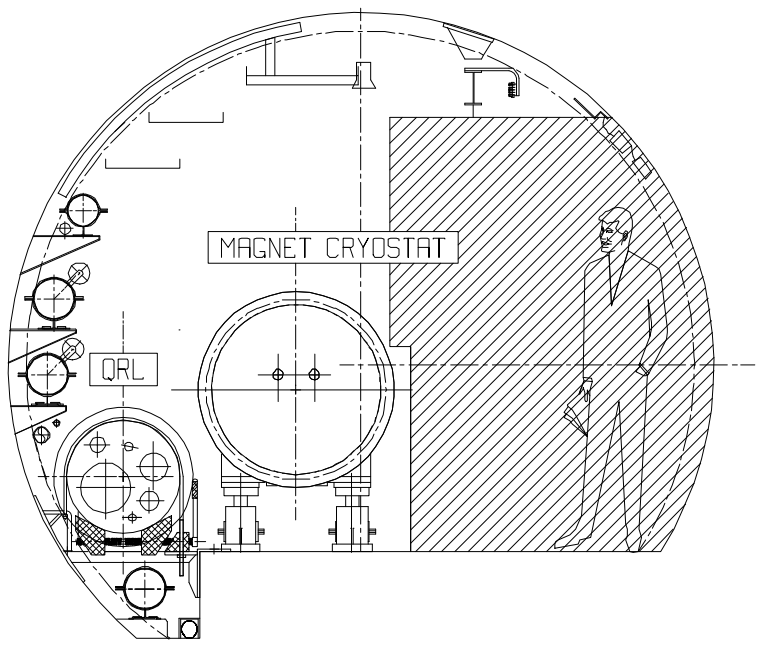

(a) with QRL service module

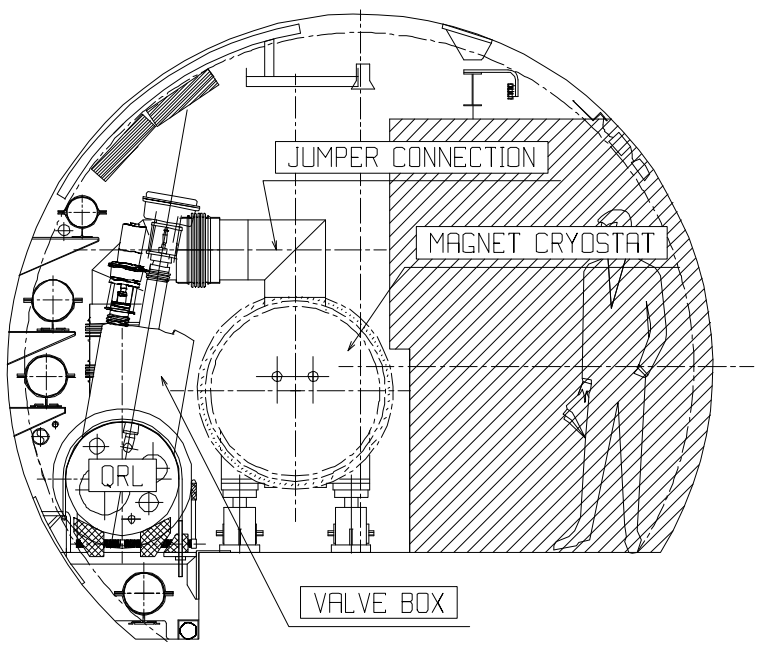

(b) with QRL pipe module

Figure 4. Standard LHC tunnel cross-sections 
The ancillary equipment has been designed at CERN and three complete sets are under construction at CERN. Up to two QRL test cells will be tested in parallel, as the very tight time schedule will not allow for testing them sequentially.

The QRL test set-up will allow for separate measurements of the heat inleaks to each header and to the Service Modules. As foreseen for the LHC machine, header C will be used as a supply line and headers B, D and F as return lines. The QRL Test Cell will not operate exactly under the same nominal conditions as the QRL supplying the LHC magnet cryostats. The main difference concerns the header pressures which for the test cell will be around 0.1 MPa for all the headers. For the operational and thermal performance assessment tests, the QRL Test Cells will be equipped with instrumentation for precision measurement of temperatures, pressures and flowrates as well as forces.

To test the three QRL test cells a test area at CERN is being built. It consists of a concrete floor $\left(130 \times 13 \mathrm{~m}^{2}\right)$, on which concrete blocks will be placed to simulate the tunnel conditions. The whole structure will be closed at each extremity and will be covered by a metallic roof.

Once the QRL test cell will be installed in the dedicated test area, the pressure test will be performed. After this test has been passed successfully, the CERN ancillary equipment will be mounted to the QRL Test Cell. After the cool-down of a Test Cell, heat inleak measurements, mechanical and alignment tests and thermal cycles will be carried out. The heat inleak measurement will then be repeated. To verify the chosen safety concept, loss of insulation vacuum will be tested as well. Finally a visual inspection of the QRL Test Cell will be done. The vacuum jacket sleeves at the assembly interfaces will be opened and the main components such as MLI, thermal shield, inner supports and headers will be inspected. The total time foreseen for testing each QRL Test Cell is about 3 months.

\section{QRL PROJECT PLANNING}

In autumn 1998 CERN adjudicated three contracts in parallel for the supply of a Preseries Test Cell in order to assess the very critical cost to performance ratio for the QRL project. The contractors are Air Liquide/France, the consortia Linde-Babcock/Germany and a group of five companies under the leadership of ABB Enertech/Switzerland. A formal Preliminary Design Review concluded this spring the detailed engineering design phase. The technical validation of the three Pre-series Test Cells at CERN is foreseen for the first half of 2000. The formal performance assessment with respect to the functional technical specification will conclude the present contracts by mid 2000. The suppliers having successfully passed this phase will then be invited to bid for the QRL series of the LHC. Installation will start in February 2002 and will end with the last commissioned sector mid 2004.

\section{ACKNOWLEDGEMENT}

We would like to acknowledge the contributions of our colleagues G. Mouron and N. Veillet of CERN. We would also like to thank our colleagues from DESY and BNL for helpful discussions. 


\section{REFERENCES}

1. L. Evans, The Large Hadron Collider Project, in: "Proc. ICEC 16", Elsevier Science, Oxford, UK (1997) 45:52.

2. M. Chorowski, W. Erdt, Ph. Lebrun, G. Riddone, L. Serio, L. Tavian, U. Wagner and R. van Weldeeren, A simplified cryogenic distribution scheme for the Large Hadron collider, in: "Adv. Cryo, Eng." 43, Plenum Press, New York, (1998), 395:402.

3. S. Claudet, Ph. Gayet, U. Wagner, Specification of four new large $4.5 \mathrm{~K}$ refrigerators for LHC, paper presented at this conference.

4. A. Bézaguet, Ph. Lebrun, L. Tavian, Performance assessment of industrial prototype cryogenic helium compressors for the Large Hadron Collider, in: "Proc. ICEC 17”, Institute of Physics Publishing, Bristol, UK (1999) 145:148.

5. Ph. Lebrun, L. Serio, L. Tavian and R. van Weelderen, Cooling strings of superconducting devices below 2 K: the helium II bayonet heat exchanger, in: “Adv. Cryo. Eng.", 43A (1998), 419:426.

6. L. Dufay, A. Perin, A. and R. van Weelderen, Characterisation of prototype superfluid helium safety relief valves for the LHC magnets, paper presented at this conference.

7. R. Losserand,-Madoux and L. Serio, Development of supercritical helium mass flow-meters for the Large Hadron Collider, paper presented at this conference.

8. P. Cruikshank et. al. , Design parameters for equipment installed in the LHC, LHC Engineering Specification LHC-PM-ES-0002.00, CERN, Geneva (1999).

9. W. Hees, R. Trant, Evaluation of electro pneumatic valve positioners for LHC cryogenics, LHC Project Note 190, CERN, Geneva (1999).

10. Ph. Lebrun, G Riddone, L. Tavian, L. and U. Wagner, Demands in refrigeration capacity for the Large Hadron Collider, in: "Proc. ICEC 16", Elsevier Science, Oxford, UK (1997), 95:98.

11. Ph. Lebrun, L. Mazzone, V. Sergo and B. Vullierme, Investigation and qualification of thermal insulation systems between $80 \mathrm{~K}$ and 4.2 K, Cryogenics 32, ICMC Supplement, 44:47 (1992). 